\title{
Are Opportunistic Pathogens Able to Sense the Weakness of Host through Specific Detection of Human Hormone?
}

\section{Olivier Lesouhaitier* and Marc Feuilloley}

Laboratory of Microbiology Signals and Microenvironment LMSM EA 4312, University of Rouen, 55 rue Saint Germain, 27000 Evreux, France

The discovery of the quorum sensing has been one of the greatest conceptual revolutions in microbiology showing that bacteria are not simple passive particles but real communicating cells. "Thinking" as bacteria, the interest to hide from the host defenses until there are insufficient number to synchronize their production of virulence factor and maximize their probability of success, is obvious. In these conditions, as bacteria frequently develop as complex communities, such as biofilms, inter-species communication and its counterpart, quorum quenching, also respond to functional contingencies. However, this is not responding to a great question: "Why, as we are regularly in contact with a multitude of micro-organisms, only some of them and apparently the same behave as pathogens?"

In the potential host, bacteria are immersed in an environment containing a multitude of communication and defense molecules and, if we keep-on "thinking" as bacteria it is of the greatest interest to detect the host response. In the presence of bacteria, immune cells respond by production of cytokines, but it was observed that pro-inflammatory cytokines act as stimulators of bacterial growth when they reach a sufficient concentration [1] suggesting the presence in bacteria of sensor systems for eukaryotes immune communication molecules. This was clearly demonstrated in Pseudomonas aeruginosa where interferon- $\gamma$ provokes the expression of a specific adhesin, the $P$. aeruginosa type-I lectin (PA-I lectin), via a quorum sensing dependent mechanism [2]. This process is essential for the pathogenic activity of the bacterium and the identification of the sensor as the $35 \mathrm{kDa}$ porin OprF, a major outer membrane protein common to a great number of $\gamma$-proteobacteria of the Pseudomonas genus, suggests that these observations should be extended to different other species. The host can also react to the presence of a bacterial invader by the synthesis of antimicrobial peptides. However, here also, the effect on bacteria is not systematically the expected one. For instance, it has been observed that on a reconstructed intestinal epithelium, bacteria such as $P$. aeruginosa or even Pseudomonas fluorescens, a species considered as a weak pathogen induce inflammatory cytokines production [3]. In this situation, intestinal Paneth cells generally react by the production of antimicrobial peptides such as defensins, but in the case of $P$. fluorescens, which is highly resistant to these molecules, the defense peptide has no effect on the bacterial growth but is sensed in some strains and boosts their virulence [4]. The mechanism supporting this process remains to be elucidated but this shows how much bacteria have adapted to respond to the host defences.

Immune and defense molecules are not the unique type of eukaryotic factors sensed by bacteria, although in that case it took a long time to admit this deep interaction with the host physiology. Since the 1930, clinicians observed that epinephrine can prompt bacterial infections [5] but this was considered as a consequence of the effect of catecholamines on the patients' physiology. I was necessary to wait for the work of Lyte \& Ernst [6] to reveal that catecholamines can act directly on bacterial virulence. These authors founded the term of "Microbial Endocrinology" to designate the effect of hormones on bacterial physiology, but even at this time the effect of catecholamines was essentially attributed to their capacity to act on bacteria as secondary siderophores. In 2000, Alverdy et al. [7] listed 39 bacterial species sen- sible to epinephrine, norepinephrine and/or dopamine but it was necessary to wait for the works of Sperandio et al. [8] and those of Clarke et al. [9] to show that catecholamines are actually detected by bacteria through a specific receptor or sensor. The sensor QseC, is a typical two component histidine sensor kinases system, showing homology in many bacterial species. This was pointing out the possible direct action of host stress hormones on bacterial virulence and, as an ill man is also a stressed man, a possible new approach to control infection.

However "Microbial Endocrinology" is not limited to catecholamines. Indeed, the list of hormones or neurohormones sensed by bacteria is wider [10]. For instance stress is also associated to the release of endogenous opioids peptides by neutrophils, then bacteria can be exposed to opioids during infection. Experimental studies revealed that in mice, stress results in the release of opioids into the intestinal lumen which directly activate the virulence of $P$. aeruginosa, leading to a disruption in the intestinal epithelial barrier. In order to explain this phenomenon, Alverdy's group showed that dynorphin, an endogenous $\kappa$-opioid receptor ligand enhances the virulence of the bacterium [11]. The original action of ligands of $\kappa$-opioid receptors on $P$. aeruginosa strongly supports the hypothesis that these micro-organisms express a specific sensor for opioid-related molecules. The physiological relevance of this hypothesis was studied by an in vivo experiment, showing that dynorphin is released into the intestinal lumen after injury and accumulates on desquamated epithelium, where it finally binds to $P$. aeruginosa [11]. More recently, it was shown that morphine itself can shift $P$. aeruginosa to a more virulent phenotype [12]. In other human diseases, such as heart failure, an increase of the family of natriuretic peptides is measured in blood, and this is used as a marker of heart dysfunction. An increase of the circulating level of two members of the natriuretic peptide family, the Brain Natriuretic Peptide (BNP) and the C-type Natriuretic Peptide (CNP) is also observed during endotoxic or septic shock [13]. It is then obvious that, during septicemia bacteria are exposed to natriuretic peptides and these peptides can modulate their behavior. This was confirmed in vitro showing that $P$. aeruginosa responds to BNP and CNP through a membrane sensor coupled to cyclase-dependent mechanism [14]. The binding of CNP on the bacterial natriuretic peptide sensor triggers an increase of exotoxin A and hydrogen cyanide production which globally enhances bacterial virulence [15]. All these data suggest that, in vivo the bacteria could detect the increase of natriuretic peptides associated to heart dysfunctions and ac-

*Corresponding author: Olivier Lesouhaitier, Laboratory of Microbiology Signals and Microenvironment (LMSM EA 4312), University of Rouen, 55 rue Saint Germain, 27000 Evreux, France, Tel : +33 2322915 42; Fax : +33 2322915 55; E-mail: olivier.lesouhait@univ-rouen.fr

Received May 04, 2012; Accepted May 05, 2012; Published May 11, 2012

Citation: Lesouhaitier O, Feuilloley M (2012) Are Opportunistic Pathogens Able to Sense the Weakness of Host through Specific Detection of Human Hormone? J Bacteriol Parasitol 3:e106. doi:10.4172/2155-9597.1000e106

Copyright: $(2012$ Lesouhaitier O, et al. This is an open-access article distributed under the terms of the Creative Commons Attribution License, which permits unrestricted use, distribution, and reproduction in any medium, provided the original author and source are credited. 
Citation: Lesouhaitier O, Feuilloley M (2012) Are Opportunistic Pathogens Able to Sense the Weakness of Host through Specific Detection of Human Hormone? J Bacteriol Parasitol 3:e106. doi:10.4172/2155-9597.1000e106

tivate their virulence program to take advantage of the host weakness.

The greatest bacterial populations of the human body are located in the intestine and on the skin which are the largest endocrine organs of the organism. These organs are also major targets of the response to stress and recent data suggest that understanding bacterial pathogenesis and intercellular communication could reveal new potential strategies to control bacteria-mediated diseases. The antibiotics strategy is somehow never promised to a definitive success as it is confronted to an over numerous and highly adaptable adversary. Interfering with bacterial virulence and/or communication is an especially compelling approach, as it is thought to apply less selection pressure on bacterial resistance mechanisms [16]. As learned from Bushido understanding and then using our weakness should be the best strategy to control bacteria.

\section{References}

1. Meduri GU (2002) Clinical review: a paradigm shift: the bidirectional effect of inflammation on bacterial growth. Clinical implications for patients with acute respiratory distress syndrome. Crit Care 6: 24-29.

2. Wu L, Estrada O, Zaborina O, Bains M, Shen L, et al. (2005) Recognition of host immune activation by Pseudomonas aeruginosa. Science 309: 774-777.

3. Madi A, Lakhdari $O$, Blottière HM, Guyard-Nicodème $M$, Le Roux K, et al. (2010) The clinical Pseudomonas fluorescens MFN1032 strain exerts cytotoxic effect on epithelial intestinal cells and induces Interleukin-8 via the AP-1 signaling pathway. BMC Microbiol 10: 215.

4. Madi A, Al-Nabhaniz Z, Orange N, Feuilloley M, Connil N (2011) Human $\beta$-defensin-2 modulates cytotoxicty of Pseudomonas fluorescens on Caco-2/ TC7 cells. $4^{\text {th }}$ FEMS Congress of European Microbiologists. Geneva, Switzerland.

5. Renaud M, Miget A (1930) Role favorisant des perturbations locales causées par l'adrenaline sur le développement des infections microbiennes. CR Seances Soc Biol Fil 103: 1052-1054.
6. Lyte M, Ernst S (1992) Catecholamine induced growth of gram negative bacteria. Life Sci 50: 203-212.

7. Alverdy J, Holbrook C, Rocha F, Seiden L, Wu RL, et al. (2000) Gut-derived sepsis occurs when the right pathogen with the right virulence genes meets the right host: evidence for in vivo virulence expression in Pseudomonas aeruginosa. Ann Surg 232: 480-489.

8. Sperandio V, Torres AG, Jarvis B, Nataro JP, Kaper JB (2003) Bacteria-host communication: the language of hormones. Proc Natl Acad Sci U S A 100: 8951-8956.

9. Clarke MB, Hughes DT, Zhu C, Boedeker EC, Sperandio V (2006) The QseC sensor kinase: a bacterial adrenergic receptor. Proc Natl Acad Sci USA 103 10420-10425.

10. Lesouhaitier O, Veron W, Chapalain A, Madi A, Blier AS, et al. (2009) Gramnegative bacterial sensors for eukaryotic signal molecules. Sensors (Basel) 9: 6967-6990.

11. Zaborina O, Lepine F, Xiao G, Valuckaite V, Chen Y, et al. (2007) Dynorphin activates quorum sensing quinolone signaling in Pseudomonas aeruginosa. PLoS Pathog 3: e35.

12. Babrowski T, Holbrook C, Moss J, Gottlieb L, Valuckaite V, et al. (2012) Pseudomonas aeruginosa virulence expression is directly activated by morphine and is capable of causing lethal gut-derived sepsis in mice during chronic morphine administration. Ann Surg 255: 386-393.

13. Post F, Weilemann LS, Messow CM, Sinning C, Münzel T (2008) B-type natriuretic peptide as a marker for sepsis-induced myocardial depression in intensive care patients. Crit Care Med 36: 3030-3037.

14. Veron W, Lesouhaitier O, Pennanec X, Rehel K, Leroux P, et al. (2007) Natriuretic peptides affect $P$ seudomonas aeruginosa and specifically modify lipopolysaccharide biosynthesis. FEBS J 274: 5852-5864.

15. Blier AS, Veron W, Bazire A, Gerault E, Taupin L, et al. (2011) C-type natriuretic peptide modulates quorum sensing molecule and toxin production in Pseudomonas aeruginosa. Microbiology 157: 1929-1944.

16. Rasko DA, Sperandio V (2010) Anti-virulence strategies to combat bacteriamediated disease. Nat Rev Drug Discov 9: 117-128. 